\title{
Development of 5000A single square pulse current standard source for microelectronic calibration
}

\author{
Liu Chong ${ }^{1, a}$, Li Jie ${ }^{2, b}$, Zhang Shan ${ }^{3, c}$, Kan Jingsong ${ }^{4, d}$ \\ ${ }^{1}$ China Electronics Standardization Institute, Bei Jing, China \\ ${ }^{2}$ China Electronics Standardization Institute, Bei Jing, China \\ ${ }^{3}$ China Electronics Standardization Institute, Bei Jing, China \\ ${ }^{4}$ China Electronics Standardization Institute, Bei Jing, China \\ aliuchong@cesi.cn, ${ }^{b}$ lijie@cesi.cn, ${ }^{2} z h a n g s h a n @ c e s i . c n,{ }^{d}$ kanjingsong@cesi.cn
}

Keywords: Power technology, distribution parameters calibration, power IGBT modules test system.

Abstract. With continuous development of high power equipment in industrial controlling area, the power levels of power semiconductor devices are also continuously increasing, and it brings many challenges to the production and testing of power semiconductor manufacturers. In this paper, the power technology at home and abroad was studied deeply, and also the distribution parameters of the hardware structure of the pulse current source were determined by means of electromagnetic transient analysis software. The design and realization method of the pulse current source were discussed in detail and the output performance and the specification of pulse current source were verified in detail. Now, the pulse current source is widely used in the calibration of the power IGBT modules test system.

\section{Introduction}

With the development of power semiconductor devices, Insulated Gate Bipolar Transistors (IGBT) were widely used in inverter circuit, the servo control system, AC motor, uninterruptible power supply, power supply, and other industrial productions in place of the MOSFET, SCR, bipolar power devices and other power semiconductors for its advantage of high input impedance, low switching loss, fast switching speed, simple control circuit and other characteristics. Now the quality performance of the IGBT modules is directly related to the quality and success of the project.

According to the MIL 750 standards, when the high power is applied to the power semiconductor devices, the test data of them are not stable because of the temperature rising in the testing process. And the DUT applied on with a long period of time of the high power can cause damage.

In order to reduce the influence of the temperature rising, just as MIL750 standards, in the National standards in China, such as GJB 128, GB 4587, and so on, pulse test method is proposed to test the parameters of the power semiconductor devices in the power semiconductors test system. That means, in the test system, single square pulse current standard source is often used to apply prescribed conditions on the DUT, and measuring unit is often used to read the response of the parameters. As for power IGBT modules, the pulse width of the current source and measuring unit is usually set as 50 micro seconds.

In China, imported power IGBT modules test system is widely used. The typical model are the TRDS series from Switzerland. For the calibration of this kind of equipment, the partial parameter method is usually adopted. Because the pulse standard current source is hard to develop, the calibration of the pulse current measurement unit is a technical difficulty. The pulse current source developed in this paper can satisfy the calibration of the pulse current measurement unit in most of the power IGBT module test equipments.

\section{System structure of the 5000A single square pulse current standard source}

The modular structure is adopted to design the 5000A single square pulse current standard source. It is composed of high voltage charging unit, charging protection unit, high voltage measurement unit, LC charge and discharge network, impedance matching unit, pulse detection unit, and monitoring control unit. The overall structure is shown in Figure 1 


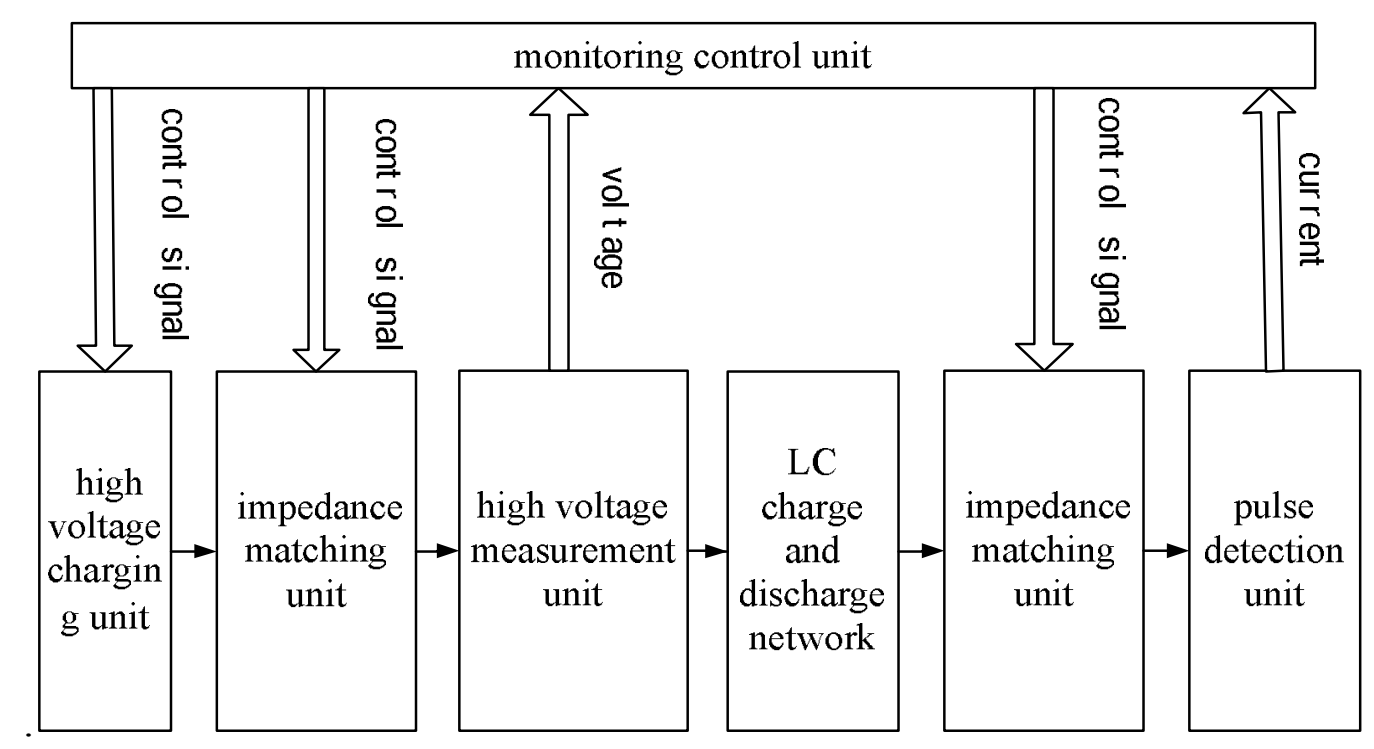

Figure 1. System structure of the standard source

The pulse current standard source mainly can be divided into two parts, hardware and software. The monitoring control unit controls the high voltage charging unit to transmit the power to the LC charging and discharging network by sending control signals. The high voltage measurement unit acquires the voltage amplitude of the LC charging and discharging network in real time and feedback it to the monitoring and control unit. When the voltage acquisition value is equal to the system setting value, the monitoring control unit controls the discharge matching unit to discharge the current, and at this point, the pulse detection unit transmits the collected pulse current signals to the monitoring and control unit to display. The monitoring control unit starts the discharge protection unit and stops the high-voltage charging unit to supply the power when the device is operated improperly or the device is broken. The monitoring control unit turns off the discharge protection unit and prepares to charge the LC charging and discharging network when the circuit breaker has been excluded.

\section{System design and Implementation}

There are two kinds of common realization models to generate single square pulse current, namely, transmission line model and capacitor charging and discharging model.

The 5000A single square pulse current standard source uses capacitor charging and discharging model to design the LC charging and discharging network. Its theoretical model is shown in Figure 2.

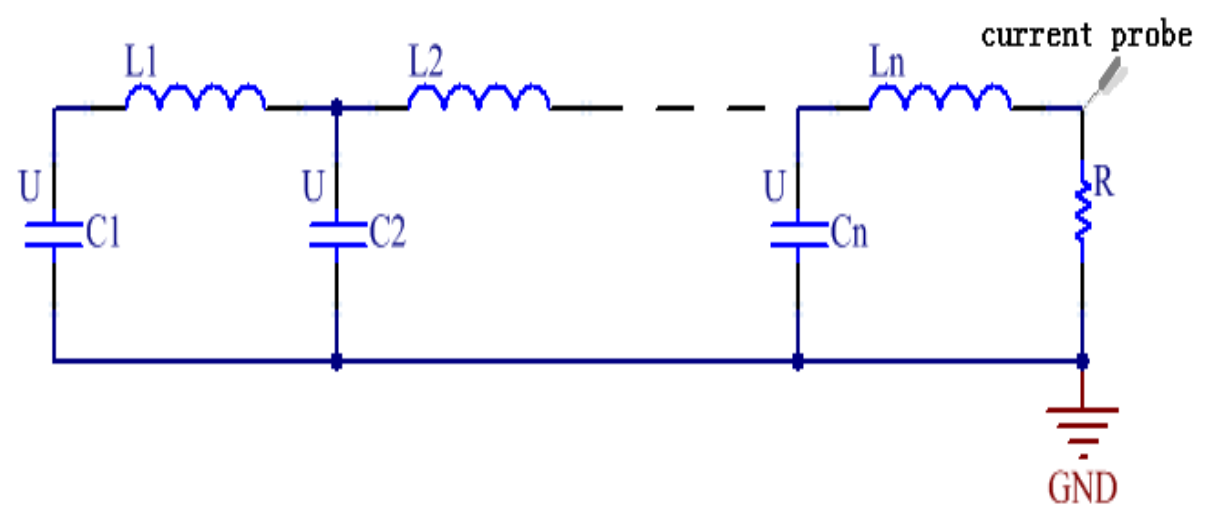

Figure 2. theoretical model of LC charging and discharging network 
According to the theoretical model characteristic data, the network parameter wave impedance $\mathrm{Z}$ can be expressed as:

$$
Z=\sqrt{\frac{L_{\Sigma}}{C_{\Sigma}}} .
$$

Among them, $L_{\Sigma}$ a represents the sum of inductance of the LC network, and $C_{\Sigma}$ represents the sum of the capacitances of the LC network. The transmission speed of $\mathrm{v}$ in the medium wave network can be expressed as:

$$
v=\frac{1}{n \sqrt{L_{\Sigma} C_{\Sigma}}}
$$

Among them, 1 represents the transmission length of the network medium wave, and $\mathrm{n}$ represents the number of the LC network order. When the load resistance $\mathrm{R}$ is equal to the wave impedance $\mathrm{Z}$, the waveform duration $\mathrm{T}$ can be expressed as:

$$
\begin{aligned}
& T=\frac{a}{v}=2 n \sqrt{L_{\Sigma} C_{\Sigma}} . \\
& T_{0.9} \approx \frac{\Varangle n-1)}{n} \sqrt{L_{\Sigma} C_{\Sigma}} .
\end{aligned}
$$

T0.9 represents the pulse width, thus the total capacitance of the LC network can be expressed as:

$$
C_{\Sigma}=\frac{n T_{0.9}}{2 R(n-1)}
$$

The total inductance of the LC network can be expressed as:

$$
L_{\Sigma}=C_{\Sigma} R^{2}
$$

The amplitude I of the single square pulse current standard source can be calculated through the following formula:

$$
I=\frac{U}{R+Z}=\frac{U}{2 R}
$$

U represents the voltage applied on the LC network.

It is expected that the maximum current amplitude of the single square pulse current standard source is $5000 \mathrm{~A}$, the last time of the waveform is $50 \mu \mathrm{s}$, and the rise time and fall time is not bigger 
than $15 \mu \mathrm{s}$. In order to get the smooth pulse current waveform, we can set $\mathrm{n}$ as 12 , the load impedance $\mathrm{R}$ can be set as $1 \Omega$, take R as $1 \Omega$ into the formula (5), the total capacitance of the LC network can be calculated out as $27.27 \mu \mathrm{F}$. and the average capacitance per chain is $2.27 \mu \mathrm{F}$. In order to obtain the amplitude 5000A of the single square pulse current standard source, according to the formula (7), the external charging voltage $U$ can be calculated out as $10000 \mathrm{~V}$. The total inductance of the chain is obtained by formula (6) as $27.27 \mu \mathrm{H}$.

On the basis of analysis of the electromagnetic transient program ATP-EMTP, the LC network model shown in Figure 2 is constructed. According to the formula (1) (7), the parameters are set reasonably and the inductance parameters of each chain are assigned, and the simulation results shown in Figure 3 are obtained.

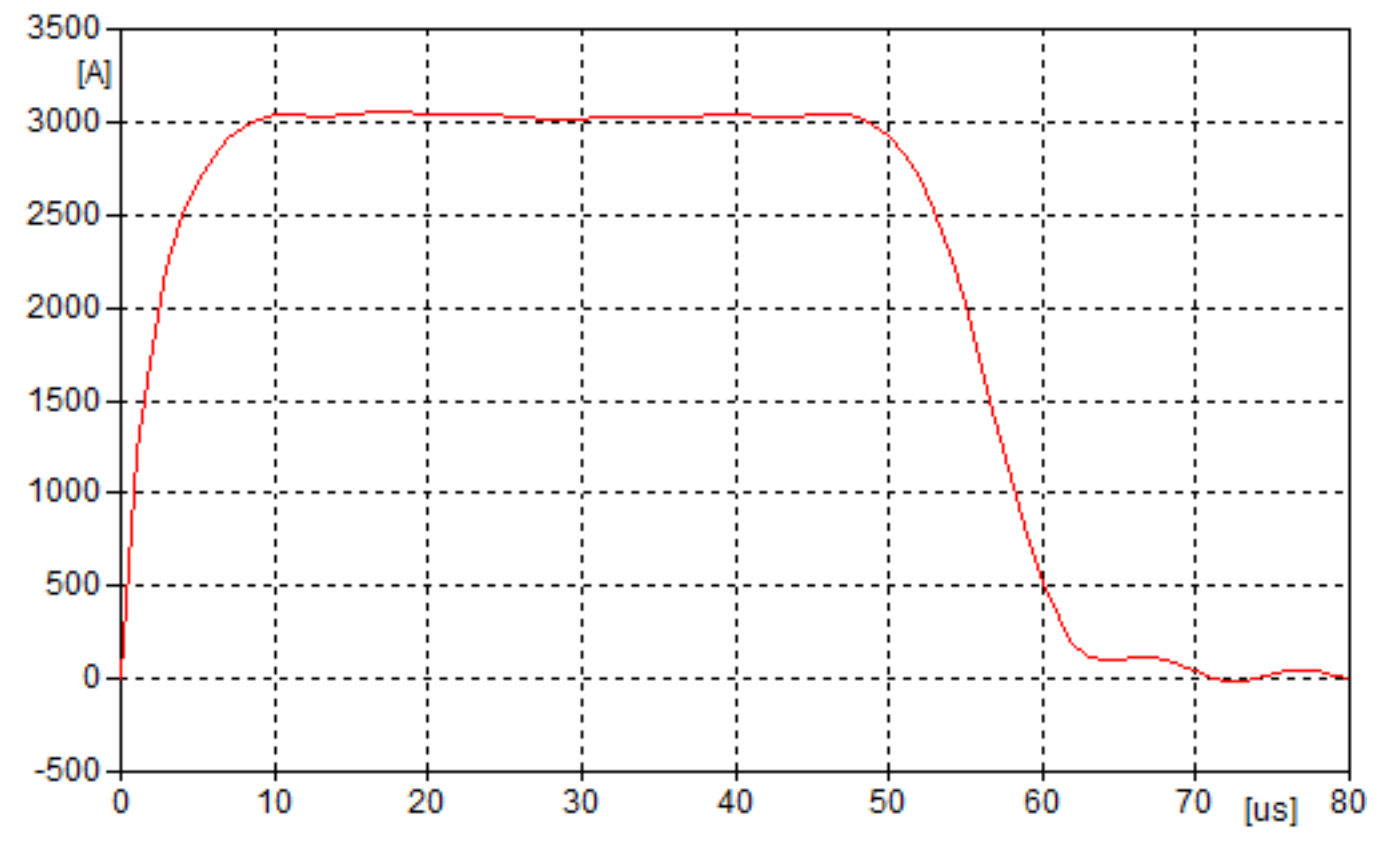

Figure 3. the simulation results

\section{Hardware design and Implementation}

The hardware part of the single square pulse current standard source realizes the function of pulse signal generation, adjustment, conversion and circuit protection. In the charging circuit, the high voltage charging unit supplies power to the LC charging and discharging network, and the DC high voltage source is customized to provide for the LC network according to the maximum amplitude of the single square pulse current standard source. By theoretical calculation, the DC voltage should be more than $10000 \mathrm{~V}$. The charging protection unit not only keeps the charging speed of the energy storage capacitors, but also ensures that the charging can be stopped in time and the discharging charge can be stopped in case of the short circuit. The high voltage detecting unit and the pulse detecting unit convert the pulse current generated by the discharge of the LC charging and discharging network into small signals respectively to the monitoring control unit. LC charging and discharging network is mainly composed of energy storage capacitor and adjustable wave inductance. The energy storage capacitor must be able to withstand a high voltage rating, and the manually adjustable wave inductance needs to adjust the square wave shape that meets the requirements of rise and fall time.

The mercury switch is selected as the key devices of the discharge switch circuit for its quickly through effects, flow capacity and small contact impedance. The load resistance value is decided by the impedance of the LC charging and discharging circuit, and the resistance of $1 \mathrm{ohm}$ should be chosen by 
theoretical calculation. The monitoring and control unit mainly realizes the functions of charging and discharging control, circuit protection and collecting voltage and current signals. The system hardware structure diagram is shown in Figure 4.

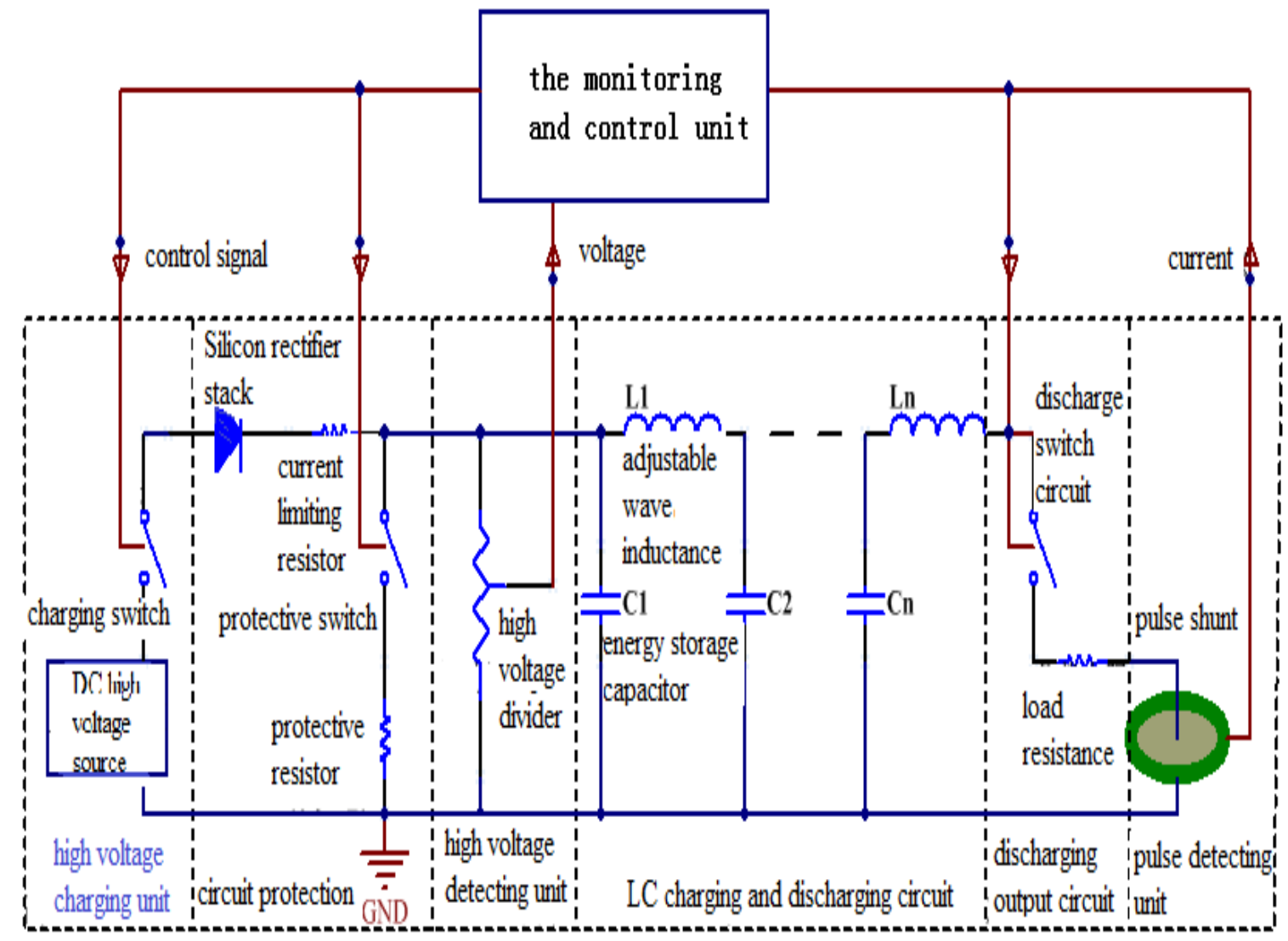

Figure 4. system hardware structure diagram

\section{Software design and Implementation}

The measurement and control software is designed on the PLC development tool CX-Programmer and the panel development tool EB8000 respectively. The former is used to design the program on OMRON PLC controller, which is mainly used to control charging and discharging switches, setting charging voltage and collecting signals. The latter designs the communication and function integration software with the PLC controller, that is, the control on the touch control interface, the program sends the corresponding control instructions to PLC and perform the corresponding control function. The control interface is shown in Figure 5. 


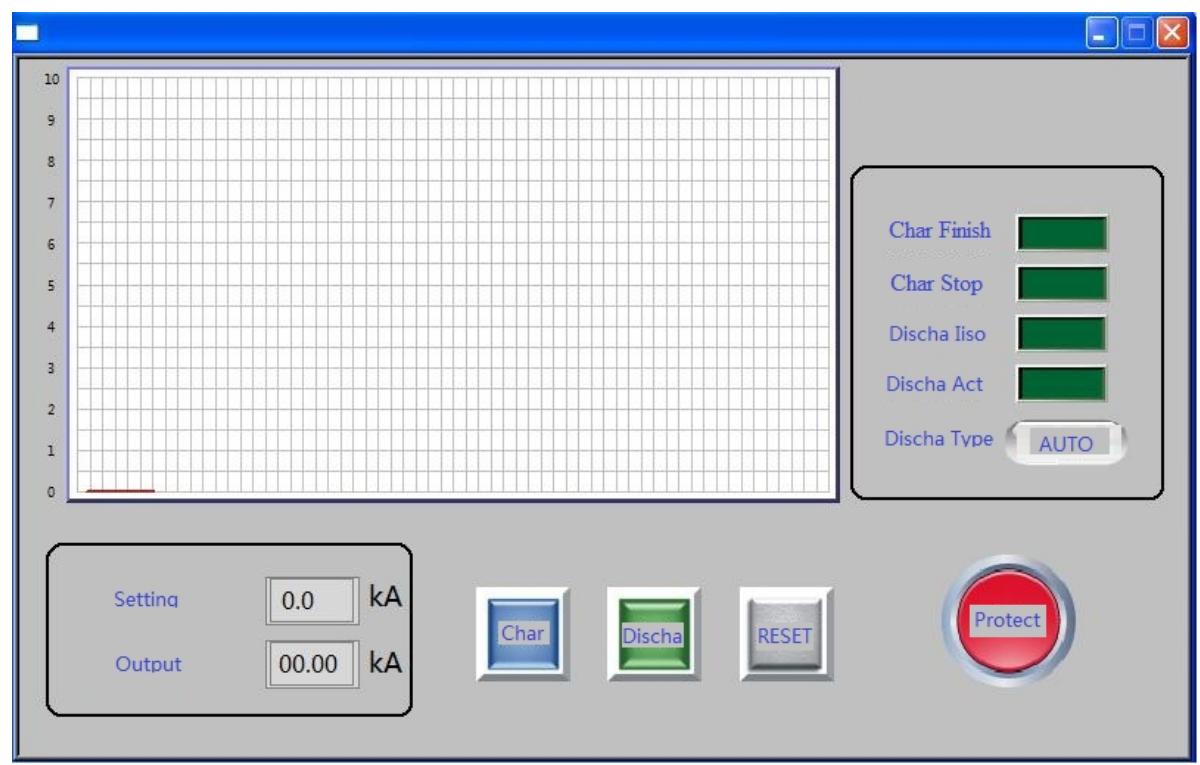

Figure 5. the control interface

All the control switches are reset first after the program is started. The user sets the output current amplitude, and then clicks the charging button. The charging switch is switched on, and the voltage and current signals are collected in real time. When the amplitude of the collected current is equal to the setting value, the charging completion state indicator lights up and indicates that the discharge is ready. After the discharge button is clicked, the discharge program is executed, and the amplitude of the current is displayed on the panel to complete the discharge process. The control program executed is shown in Figure 6.

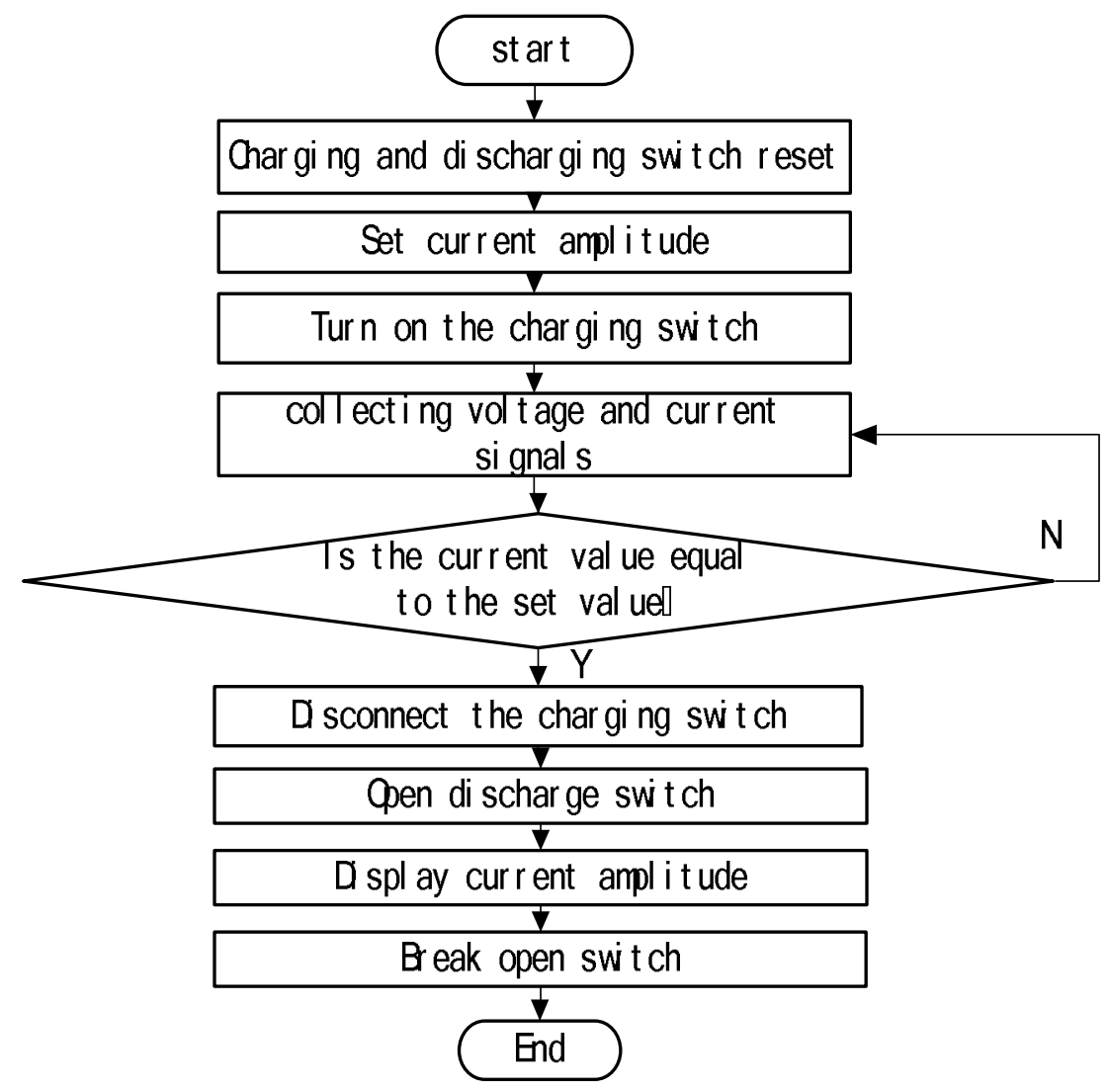

Figure 6. software control flow chart 


\section{Performance verification of the 5000A single square pulse current standard source}

The maximum current amplitude of the current standard source is $5000 \mathrm{~A}$, it can not be measured directly for its single and high characteristics, so it can be measured by data acquisition equipment through shunts or coils. The test experiments is fulfilled by following instruments, model 4418 (0.001V/A) pearson coil, and NI data acquisition with the model PXI-5122. The pearson coil has the advantages of fast response time, low signal distortion and no position limitation. Through data acquisition NI PXI-5122, the waveform can be captured and displayed, and the output amplitude value of the standard current source can be obtained by calculation. The performance verification connection is shown in Figure 7.

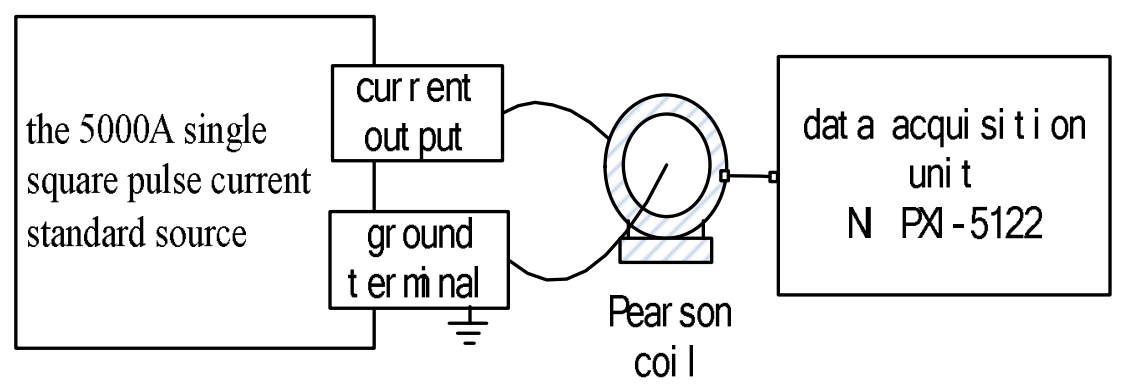

Figure 7. performance verification connection

When the output current amplitude is $5000 \mathrm{~A}$, through performance verification tests, the measured waveform is shown as shown in Figure 8.

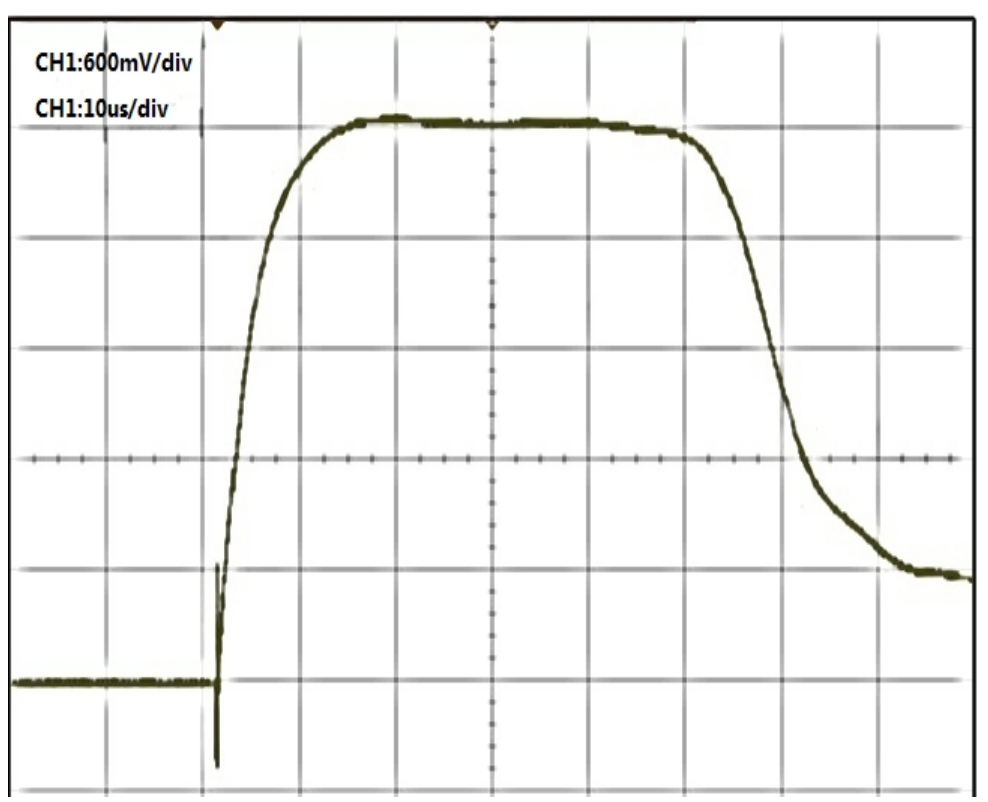

Figure 8. measured waveform when the output current amplitude is 5000A

In the verification of the output accuracy of the 5000A single square pulse current standard source, test results is selected as the average value in the area of $1 / 3$ of the stable middle part for the waveform. The measurement results of the single square pulse current standard source are shown in Table 1 . 
Table 1. measurement results

\begin{tabular}{|c|c|c|c|c|}
\hline No. & Setting Value & $\begin{array}{c}\text { Average value of } \\
\text { pulse voltage } \\
\text { measurement }\end{array}$ & $\begin{array}{c}\text { Average value of } \\
\text { the standard } \\
\text { source }\end{array}$ & Error \\
\hline 1 & $20 \mathrm{~A}$ & $0.01992 \mathrm{~V}$ & $19.92 \mathrm{~A}$ & $0.4 \%$ \\
\hline 2 & $100 \mathrm{~A}$ & $0.09982 \mathrm{~V}$ & $99.82 \mathrm{~A}$ & $0.18 \%$ \\
\hline 3 & $500 \mathrm{~A}$ & $0.4986 \mathrm{~V}$ & $498.6 \mathrm{~A}$ & $0.28 \%$ \\
\hline 4 & $1000 \mathrm{~A}$ & $0.9961 \mathrm{~V}$ & $996.1 \mathrm{~A}$ & $0.39 \%$ \\
\hline 5 & $2000 \mathrm{~A}$ & $1.987 \mathrm{~V}$ & $1987 \mathrm{~A}$ & $0.65 \%$ \\
\hline 6 & $5000 \mathrm{~A}$ & $4.982 \mathrm{~V}$ & $4982 \mathrm{~A}$ & $0.36 \%$ \\
\hline
\end{tabular}

From Figure 8. and Table 1, it can see that the output waveform of the single square pulse current standard source satisfies the square wave characteristic, and the specification is within $1 \%$.

\section{Conclusions}

In this paper, the calibration technology of power IGBT module test equipment is studied deeply, and the 5000A pulse standard current source is used to calibrate. This paper introduces in detail the principle of pulse generator and the design of software and hardware, the actual test results show that the standard current source is stable and the specification meets the calibration needs of the power IGBT module test equipments.

\section{Acknowledgements}

This work was financially supported by the China Electronics Stadardization Institute.(100176).

\section{References}

[1] Zhang Yufeng,Liu Chong,Yu Lihong,in:Research on Calibration Technology of power IGBT module test equipment, December 2014 of Electronics today.

[2] Chen Jingliang, Yao Xueling. Pulse current technology [M]. Xi An, China: Xi'an Jiao Tong University press, 2008:57 65.

[3] Zhang Renyu, Chen Changyu. High voltage experimental technique [M]. Bei Jing, China : Tsinghua university press, 2009: 188 190.

[4] Liu Chong, Yu Lihong. Research on Amplitude Calibration of High Pulse Current Source of Semiconductor Device Test System.[C] //IEEE Conference on Electronic Measurement \& Instruments. 2011: II 232 235. 